\title{
Criminologie
}

\section{L'injonction thérapeutique à l'égard des toxicomanes : comparaison des systèmes français et québécois}

\section{François Facy, Serge Brochu et Françoise Simon}

Volume 29, numéro 2, automne 1996

L'homicide conjugal au Canada

URI : https://id.erudit.org/iderudit/017392ar

DOI : https://doi.org/10.7202/017392ar

Aller au sommaire du numéro

\section{Éditeur(s)}

Les Presses de l'Université de Montréal

ISSN

0316-0041 (imprimé)

1492-1367 (numérique)

Découvrir la revue

Citer cet article

Facy, F., Brochu, S. \& Simon, F. (1996). L'injonction thérapeutique à l'égard des toxicomanes : comparaison des systèmes français et québécois. Criminologie, 29(2), 115-140. https://doi.org/10.7202/017392ar
Résumé de l'article

In Canada, the total amount of accusations, in compliance with the laws on drugs, show a slight but constant progression. More than half of the sentences for drug-linked offenses have lead to incarceration, a third of the people sentenced for a simple drug possession have been jailed. The relevance of these sentences, making the Canadian detention centres populated with more drug addicts than the rehabilitation centres, is questionable.

In France, legislation is mostly based on the December 31st, 1970 law: drug use and trafficking are severely punished. However, for a first arrest, the prosecutor can enjoin the arrestee to treatment: this is what is called therapeutic injunction. Even though therapeutic injunction spares substance users from a jail term, some limitations are encountered this measure is selective, based on social and judicial criteria, making it more forgiving than the average characteristics of drug addicts, it is only accepted and applied by half of the subjects. A specific inquiry has been done in a department in the Paris region, where drug addiction activity is abundant, and suggested a preventive role for the injunction in cases of recidivism. Global appreciation of the therapeutic injunction remains nuanced in a context of new measures in drug addiction, but it translates into the increasing necessity to introduce professional networks between the judicial and sanitary systems, intended for a young population dealing with the modern difficulties of social adaptation and high risk behaviour.
Ce document est protégé par la loi sur le droit d'auteur. L'utilisation des services d'Érudit (y compris la reproduction) est assujettie à sa politique d'utilisation que vous pouvez consulter en ligne.

https://apropos.erudit.org/fr/usagers/politique-dutilisation/ 
In Canada, the total amount of accusations, in compliance with the laws on drugs, show a slight but constant progression. More than half of the sentences for drug-linked offenses have lead to incarceration. a third of the people sentenced for a simple drug possession have been jailed. The relevance of these sentences, making the Canadian detention centres populated with more drug addicts than the rehabilitation centres, is questionable.

In France, legislation is mostly based on the December 31st, 1970 law: drug use and trafficking are severely punished. However, for a first arrest, the prosecutor can enjoin the arrestee to treatment: this is what is called therapeutic injunction. Even though therapeutic injunction spares substance users from a jail term, some limitations are encountered this measure is selective, based on social and judicial criteria, making it more forgiving than the average characteristics of drug addicts, it is only accepted and applied by half of the subjects. A specific inquiry has been done in a department in the Paris region, where drug addiction activity is abundant, and suggested a preventive role for the injunction in cases of recidivism. Global appreciation of the therapeutic injunction remains nuanced in a context of new measures in drug addiction, but it translates into the increasing necessity to introduce professional networks between the judicial and sanitary systems, intended for a young population dealing with the modern difficulties of social adaptation and high risk behaviour.

\section{1 - LA SITUATION EN FRANCE}

Dans les années 1970, face à l'augmentation des comportements toxicomaniaques, le système français de réponses sanitaires et sociales s'est peu à peu installé, en fonction de la législation, du code de la santé publique et des implications des professionnels de la santé, du champ médical et social.

1. Directeur de Recherche, INSERM U.302, 44, Chemin de Ronde, 78110 Le Vésinet, France.

2. Professeur agrégé, École de criminologie et chercheur associé, Centre intemational de criminologie comparée, Université de Montréal, C. P. 6128, Succ. Centreville, Montréal, Québec, H3C 3J7, Canada.

3. Psychologue DDASS, Hauts-de-Seine. 
On constate ainsi une grande spécificité du système de prise en charge en France par rapport à d'autres pays voisins, de niveau économique et culturel équivalent et d'organisation socio-politique comparable (Leroy, 1991 ; Boucaud, 1990 ; Porter, 1988).

La législation touchant au trafic et à l'usage des «substances vénéneuses » est de plus en plus complexe. Elle se base principalement sur la loi du 31 décembre 1970: le trafic est sévèrement réprimé, l'usage de stupéfiants l'est aussi. Les utilisateurs sont passibles d'une peine de 2 mois à 1 an de prison. Lors d'une première arrestation, le procureur peut enjoindre à l'usager de se soumettre à un traitement : c'est l'injonction thérapeutique.

Sur le plan légal, il s'agit d'une disposition particulière de la loi du $31 / 12 / 70$ régissant la toxicomanie : elle permet au parquet de transférer à l'autorité sanitaire (Direction départementale des affaires sanitaires et sociales - DDASS) les personnes qui lui sont présentées pour usage de produits toxiques illicites après interpellation des services de police. Sont écartés de cette mesure tous ceux à l'encontre desquels est retenue la présomption de trafic. Il s'agit ainsi de personnes faisant un usage occasionnel ou régulier de substances toxiques interdites (Parquet, 1988; Delobel, 1990; Leblanc, 1991).

Les personnes, après interpellation par un des différents services de police et éventuellement après garde à vue, comparaissent libres, sur convocation, dans le cabinet du substitut; le magistrat leur soumet deux options : suivi thérapeutique ou poursuites judiciaires. Il indique également que si le contrat sanitaire est correctement rempli, les poursuites (qui ne sont que suspendues) ne reprendront pas. Le dossier sera classé sans suite, ce qui signifie, sur le plan pratique, qu'il n'y aura pas d'inscription au casier judiciaire. $C$ 'est une disposition qui permet de ne pas laisser de trace judiciaire si le résultat de la mesure est considéré comme positif (fig. 1), (Fauconnet, 1986).

Un entretien confidentiel est organisé par le DDASS, au cours duquel est établi un bilan d'évaluation et d'orientation. Le service n'assure en aucun cas le suivi thérapeutique, mais seulement le suivi administratif. Selon divers critères, les sujets peuvent être orientés vers :

- des associations spécialisées du département;

- des centres médico-psychologiques (dépendant d'hôpitaux);

- des médecins généralistes.

Dans les trois hypothèses, la prise en charge est gratuite, quelle que soit sa durée et sa nature.

Les institutions chargées du suivi communiquent (sans obligation toutefois) au service de la DDASS concerné les dates des consultations ou 
entretiens, et indiquent si une hospitalisation a été réalisée en vue d'un sevrage (Sabatini, 1990).

Au terme du délai imparti par le parquet ( $3-4$ mois), le magistrat est informé par le service DDASS de la suite donnée à l'injonction. Si elle a été favorable, le dossier est classé sans suite. Dans le cas contraire, les poursuites reprennent et aboutissent souvent à une peine avec sursis, assortie d'une mesure de mise à l'épreuve (Ligier, 1980).

Parmi les informations disponibles sur les différents pôles d'activités auprès des toxicomanes, l'indicateur de l'injonction thérapeutique est particulièrement significatif de l'organisation sociale et du fonctionnement des équipes de justice et de santé, face à une population par essence fluctuante, difficile à cerner et à suivre.

Différentes statistiques sont tenues par le ministère de la Justice. Actuellement, on estime à $15 \%$ l'importance des toxicomanes incarcérés pour ILS ${ }^{4}$ ou pour d'autres délits (pour un total de 57000 personnes incarcérées). La mesure d'injonction thérapeutique a été très peu utilisée dans les années 70, elle est fortement relancée depuis 1992. En 1993, 6342 injonctions thérapeutiques sont prononcées, alors que le nombre d'interpellations pour usage (et usage revente) de drogues s'élève à 16 573. Par ailleurs, des études épidémiologiques reflètent les préoccupations de la santé des populations (incarcérées ou en injonction) et décrivent leurs caractéristiques et leur état de santé (Facy, 1993).

Deux enquêtes particulières ont été réalisées depuis 1990, au niveau général đes DDASS, qui sont chargées d'appliquer les injonctions thérapeutiques et de vérifier le suivi des patients adressés aux équipes spécialisées, et au niveau de la DDASS des Hauts-de-Seine en collaboration avec le parquet de Nanterre, en région parisienne.

\subsection{Enquête nationale auprès des DDASS : mesure de l'application de l'injonction}

Pour apprécier le volume des activités dans chaque département en relation avec les mesures d'injonction thérapeutique, un questionnaire est adressé à chaque DDASS, où sont demandés : des données quantitatives sur le nombre de sujets concernés et suivis, des aspects budgétaires et des modalités qualitatives de prises en charge et de fonctionnement des équipes.

Pour 1990, 86 départements ont répondu à l'enquête. Il faut remarquer que la diversité des réponses reflète à la fois des situations extrêmement variables mais aussi les difficultés internes à distinguer des activités qui,

4. ILS : infractions à la législation sur les stupéfiants. 
bien souvent, s'inscrivent dans un contexte plus global de fonctionnement et d'intervention des DDASS.

\subsubsection{Volume des injonctions}

La moyenne des injonctions prononcées par le procureur a fortement augmenté en 1988, depuis elle est stable : 42 en 1990 pour 86 départements. La variabilité est très grande entre les départements. Le nombre d'injonctions prononcées va de 0 à 580 .

L'activité de la DDASS porte en moyenne sur une quarantaine de personnes qui se sont présentées en un an.

Parmi elles, 6 sur 10 ont terminé le processus de l'injonction, 1 sur 5 est en cours d'injonction, 1 sur 9 est perdue de vue.

Ce dernier chiffre semble avoir baissé depuis 1989 , ce qui pourrait refléter une plus grande efficacité des services ou une meilleure adaptation des sujets; cependant, les effectifs sont petits et incitent à la prudence dans l'interprétation.

L'appréciation des DDASS par rapport à la connaissance de la fréquentation du système spécialisé en toxicomanie par les sujets qu'ils reçoivent est intéressante, dans la mesure où elle reflète ce que les patients rapportent de leurs itinéraires antérieurs.

Pour 61 départements en moyenne, 59 toxicomanes sur 100 présentés pour injonction thérapeutique n'ont pas eu de contact antérieur avec les centres spécialisés.

\section{Tableau 1}

Nombre moyen de personnes sous injonction pour 86 đépartements

- qui se sont présentées à la DDASS :

$\begin{array}{r}1989 \\ 1990 \\ \hline\end{array}$

parmi elies :

- ayant terminé le processus de l'injonction :

1989

1990

- en cours d'injonction :

1989

4

1990

7

- perdues de vue :

1989 


\subsubsection{Modalités des interventions}

La durée moyenne de suivi d'une injonction est de 178 jours, elle est importante mais très variable, avec un écart-type de 141 , montrant ainsi les difficultés pour le personnel d'une structure face à une activité volumineuse mais fluctuante, avec des pics, suivant les cas.

Pour 76 départements ayant répondu, l'autorité sanitaire fait procéder dans $74 \%$ des cas à l'examen médical et dans $41 \%$ des cas à l'enquête sociale prévus dans l'article L.355-15 (Fauconnet, 1986).

La DDASS prend contact avec le responsable de la prise en charge dans la grande majorité des cas : $94 \%$ des 73 départements ayant répondu.

\subsubsection{Difficultés}

Les difficultés d'application des articles sont signalées dans un grand nombre de départements : $41 \%$ au total des 86 DDASS.

La nature des difficultés n'est pas suffisamment précisée dans le questionnaire pour pouvoir donner lieu à une analyse précise de l'articulation des DDASS avec les autres structures intervenant dans l'application de l'injonction thérapeutique.

Dans certains cas, elles sont en relation avec l'absence de structures spécialisées au niveau du département alors qu'à l'inverse, d'autres difficultés peuvent être suscitées par une trop grande spécialisation de professionnels, le lieu hautement spécialisé exerçant de fortes contraintes pour la prise en charge (Delpuech, 1989).

L'étude nationale a permis de montrer la diversité des applications de l'injonction thérapeutique suivant les départements, révélatrice des différences au niveau des activités de soins en général auprès des toxicomanes, mais aussi des différences des pratiques judiciaires. Pour approfondir la connaissance de l'application de l'injonction et son efficacité, une étude plus restreinte a été entreprise sur un département de la région parisienne où existe une bonne corrélation entre les recours aux centres de soins en général et les mesures d'injonction (ORS, Île-de-France, 1991).

\subsection{Enquête réalisée en région parisienne : efficacité de la mesure}

\subsubsection{Méthodologie}

L'objectif de cette enquête est de comparer l'évolution du cheminement des usagers de stupéfiants ayant répondu à une mesure d'injonction thérapeutique et de ceux qui l'ont ignorée. 
Tableau 2

Situation moyenne des 41 départements ayant plus de 10 injonctions

\begin{tabular}{lcc}
\hline Activités & $\begin{array}{c}\text { Effectif } \\
\text { moyen }\end{array}$ & $\begin{array}{c}\text { Nombre de } \\
\text { départements } \\
\text { concernés }\end{array}$ \\
\hline 一 Injonctions prononcées dans le département & 86 & 41 \\
- Injonctions reçues de l'extérieur & 7 & 41 \\
- Injonctions transmises vers l'extérieur & 8 & 41 \\
- Personnes présentées à la DDASS & 78 & 41 \\
- Personnes perdues de vue & 8 & 41 \\
- Part des sujets en 1re consultation pour & $64 \%$ & 38 \\
toxicomanie & 8 & 34 \\
- Signalements & 167 & 37 \\
Moyenne de suivi (en jours) & $73 \%$ & 41 \\
Examen médical* & $44 \%$ & 41 \\
Enquête sociale & $95 \%$ & 41 \\
Contact DDASS et responsable de prise en \\
charge
\end{tabular}

* Les pratiques sont notées dans la proportion des départements concernés et ayant répondu.

L'échantillon retenu pour l'enquête porte sur 328 personnes qui, convoquées, se sont rendues effectivement au parquet et aux services de la DDASS pour que soit prononcée et mise en place à leur égard la mesure d'injonction.

Cet échantillon englobe tous les usagers vus entre le $1^{\text {er }}$ octobre 1988 et le 30 septembre 1989.

Un groupe pluridisciplinaire composé de magistrats, de cliniciens et de chercheurs a confronté des pratiques professionnelles et des interrogations globales, avant d'élaborer un recueil de données épidémiologiques.

L'étude menée a pu être établie essentiellement à partir de quatre sources d'informations:

Les registres des magistrats de la section des stupéfiants au parquet de Nanterre, documents dans lesquels figurent l'identité et les dates de comparution au cabinet du substitut des personnes ayant été interpellées pour «usage illicite de stupéfiants » et autorisées à bénéficier de la mesure d'injonction thérapeutique après avis du magistrat. Y est également transcrit leur acceptation de la mesure, étant observé que peu de refus sont enregistrés. 
Figure 1

Articulation des services dans l'injonction therrapeutique

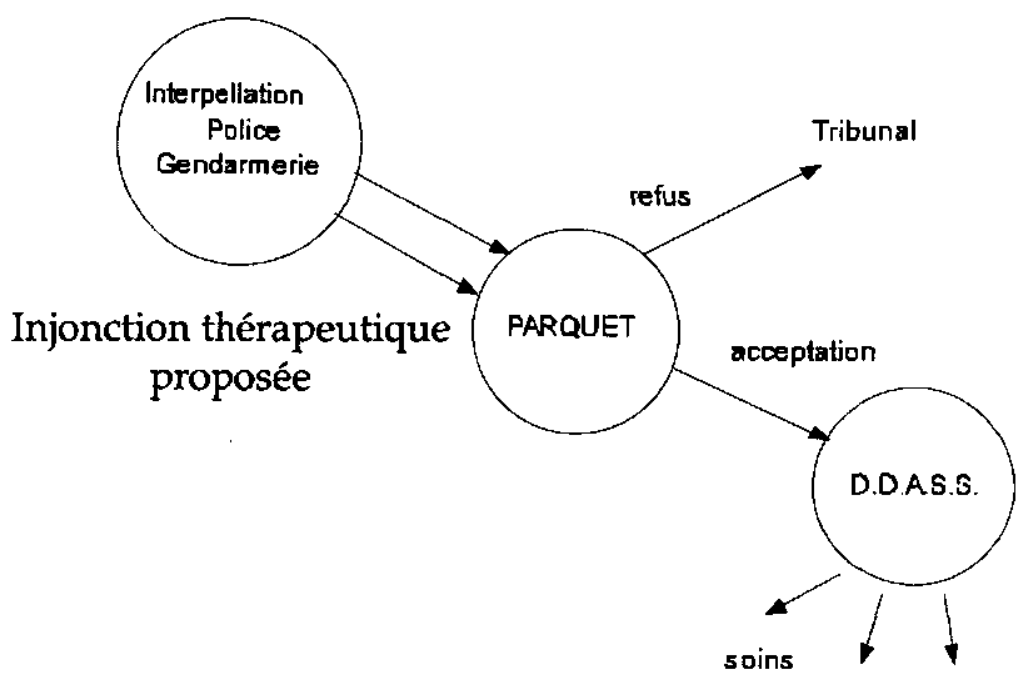

Le système informatique du tribunal de grande instance

Tous les usagers de stupéfiants ayant fait l'objet de procédure dans le ressort du T.G.I. ${ }^{5}$ ou domiciliés dans ce ressort sont portés en mémoire et l'enregistrement de ces affaires consiitue un outil utile à la présente étude.

Les cahiers de la DDASS

Au cours de l'entretien avec l'usager, la DDASS a pu recueillir un certain nombre d'informations telles que: identité, nationalité, parcours scolaire et professionnel de l'usager, contexte familial et indications spécifiques à son parcours toxicomaniaque.

Les casiers judiciaires

Il s'agit des bulletins $n^{\circ} 1$ des casiers judiciaires délivrés aux seules autorités judiciaires. Ces bulletins visés aux articles 768 et suivants du code de procédure pénale révèlent toutes les condamnations définitives prononcées à l'encontre d'un individu, à l'exception des condamnations effacées par une amnistie, réhabilitations de plein droit ou judiciaires, des condamnations prononcées depuis plus de quarante ans non suivies d'une nouvelle condamnation à une peine criminelle ou correctionnelle, des décisions

5. Tribunal de grande instance. 
prises à l'égard de mineurs de 18 ans qui après un délai de 3 ans et sur requête de l'intéressé peuvent être supprimées du casier.

\section{Figure 2}

Constitution de l'échantillon

Nombre de sujets considérés

\section{Pertes}

registres incomplets

casiers judiciaires incomplets

dossiers documentés 103

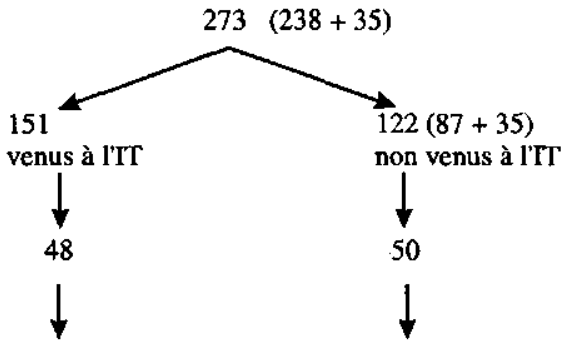

72 dossiers documentés

Le nombre total de sujets étudiés pour l'enquête est de 175. Les deux groupes obtenus finalement sont comparés par les méthodes statistiques classiques : chi-deux et test $\mathrm{t}$.

\subsubsection{Caractéristiques des sujets}

L'échantillon des usagers s'êtant présentés à l'injonction thérapeutique est caractérisé par :

- une majorité d'hommes ( $89 \%)$;

- des catégories socioprofessionnelles diverses: $30 \%$ inactifs, $23 \%$ ouvriers, $27 \%$ employés ;

- $59 \%$ ont été interpellés à Nanterre et $19 \%$ à Paris (dans ce dernier cas, les informations sur les usagers venus ou non venus ont été plus restreintes).

Parmi les 175 sujets s'étant présentés ou non à l'injonction thérapeutique, on constate que :

- $93 \%$ sont usagers d'héroïne ;

- $2 \%$ sont usagers de cocaìne;

- $5 \%$ sont usagers de cannabis.

Il faut expliquer le pourcentage très faible des usagers de cannabis par une politique du Parquet de Nanterre qui, jusqu'en janvier 1991, n'incluait pas dans les propositions à l'injonction thérapeutique les usagers de cannabis, préférant leur adresser un simple avertissement. ̇̀ compter de cette 
date, une politique nouvelle a été adoptée, visant à admettre au bénéfice de l'injonction thérapeutique tout usager de cannabis âgé de 18 à 21 ans, et ce, dans l'optique d'une poursuite de la prise en charge des mineurs usagers de cannabis.

Parmi les 175 sujets convoqués, $59 \%$ (soit 103) se sont présentés pour une mesure d'injonction thérapeutique, et $41 \%$ (soit 72 ) ne sont pas venus.

Parmi les premiers, 94 sur 103 ont suivi l'obligation de l'injonction thérapeutique et à l'issue d'un délai moyen de 3 mois, ont vu leur affaire classée.

Les autres $(6 \%)$ venus à la convocation pour une injonction thérapeutique et qui avaient accepté cette mesure ont été condamnés pour non-respect du contrat passé avec le magistrat d'une part et la DDASS d'autre part.

L'étude menée a permis de confirmer que le seul critère de sélection retenu pour faire bénéficier ou non un sujet interpellé de la mesure tendant à une injonction est $l$ 'usage de stupéfiants.

On constate que $2 \%$ des personnes interpellées ont commis un autre délit au moment de l'injonction.

Il est également établi qu'à la date de l'injonction prise par le parquet, la moitié des usagers n'ont aucun passé judiciaire : leur casier judiciaire ne mentionne aucune condamnation (tous délits confondus).

Quant à ceux qui ont connu une condamnation antérieure, 54\% l'ont eue à Nanterre, $22 \%$ à Paris.

Enfin, parmi ceux condamnés après la venue au parquet pour se voir signifier une injonction thérapeutique, $50 \%$ auront une seule condamnation ultérieure, et $15 \%$ en auront 3 et plus.

Il convient toutefois pour apprécier parfaitement ces statistiques, de rappeler que l'étude couvre une période allant de septembre 1988 à octobre 1989 et que l'examen des casiers de chaque usager s'arrête en septembre 1991.

En conséquence, la période retenue pour l'examen des casiers des sujets vus en septembre 1988 a été de 3 ans, tandis que celle des sujets étudiés en 1989 a porté sur une durée de 2 ans.

De plus, il faut souligner que les informations peuvent s'avérer incomplètes en fonction de la gestion de fichier des casiers et, par la suite, les données obtenues sont minimales. 
Tableau 3

Étude des personnes convoquées au parquet pour injonction thérapeutique

\begin{tabular}{|c|c|c|c|c|c|c|c|}
\hline \multirow{2}{*}{\multicolumn{2}{|c|}{ Moyenne d'âge à l'injonction }} & \multicolumn{3}{|c|}{$\begin{array}{l}\text { Sujets s'étant } \\
\text { présentés } \\
n=103\end{array}$} & \multicolumn{3}{|c|}{$\begin{array}{l}\text { Sujets ne s'étant } \\
\text { pas présentés } \\
\mathbf{n}=72\end{array}$} \\
\hline & & 25 an & & & 25 ans & & \\
\hline \multirow[t]{2}{*}{ Sexe } & Masculin & $92 \%$ & & & $85 \%$ & & \\
\hline & Féminin & $8 \%$ & & & $15 \%$ & & \\
\hline \multirow[t]{2}{*}{ Nationalité } & Française & $85 \%$ & & & & & \\
\hline & Étrangère & $15 \%$ & & & & & \\
\hline \multirow{3}{*}{$\begin{array}{l}\text { Catégorie } \\
\text { Socio- } \\
\text { professionnelle }\end{array}$} & Prof. diverses & $16 \%$ & & & & & \\
\hline & Ouvriers & $23 \%$ & & & Incon & & \\
\hline & Employés & $27 \%$ & & & & & \\
\hline \multirow{5}{*}{ Produits } & Étudiants & $4 \%$ & & & & & \\
\hline & Inactifs & $30 \%$ & & & & & \\
\hline & Héroïne & $93 \%$ & & & & & \\
\hline & Cocaine & $2 \%$ & & & & & \\
\hline & Cannabis & $5 \%$ & & & & & \\
\hline \multicolumn{2}{|l|}{ Suite favorable } & $91 \%$ & & & & & \\
\hline \multicolumn{2}{|l|}{ Classé sans suite } & $4 \%$ & & & & & \\
\hline \multicolumn{2}{|c|}{ Condamnation pour non-respect } & $5 \%$ & & & $1 \%$ & & \\
\hline \multirow[t]{3}{*}{ Autre délit } & Amendes etou sursis & 3 & & & 4 & & \\
\hline & Sursis \& MAE ${ }^{1}$ & 4 & & & 22 & & \\
\hline & Prison & 1 & & & 16 & & \\
\hline \multicolumn{2}{|c|}{ Infractions commises avant l'IT *** } & $38 \%$ & 0,7 & *** & $74 \%$ & 1,6 & $* * *$ \\
\hline \multicolumn{2}{|c|}{ Infractions commises après l'IT** } & $45 \%$ & 0,8 & $* *$ & $61 \%$ & 1,4 & $* *$ \\
\hline
\end{tabular}

1 MAE : mise à l'épreuve (probation).

* $\quad \mathrm{p}<0,05$.

$* * \quad p<0,01$.

$* * * \quad \mathrm{p}<0,001$. 


\section{Tableau 4}

Parcours judiciaire des personnes convoquées pour injonction therapeutique

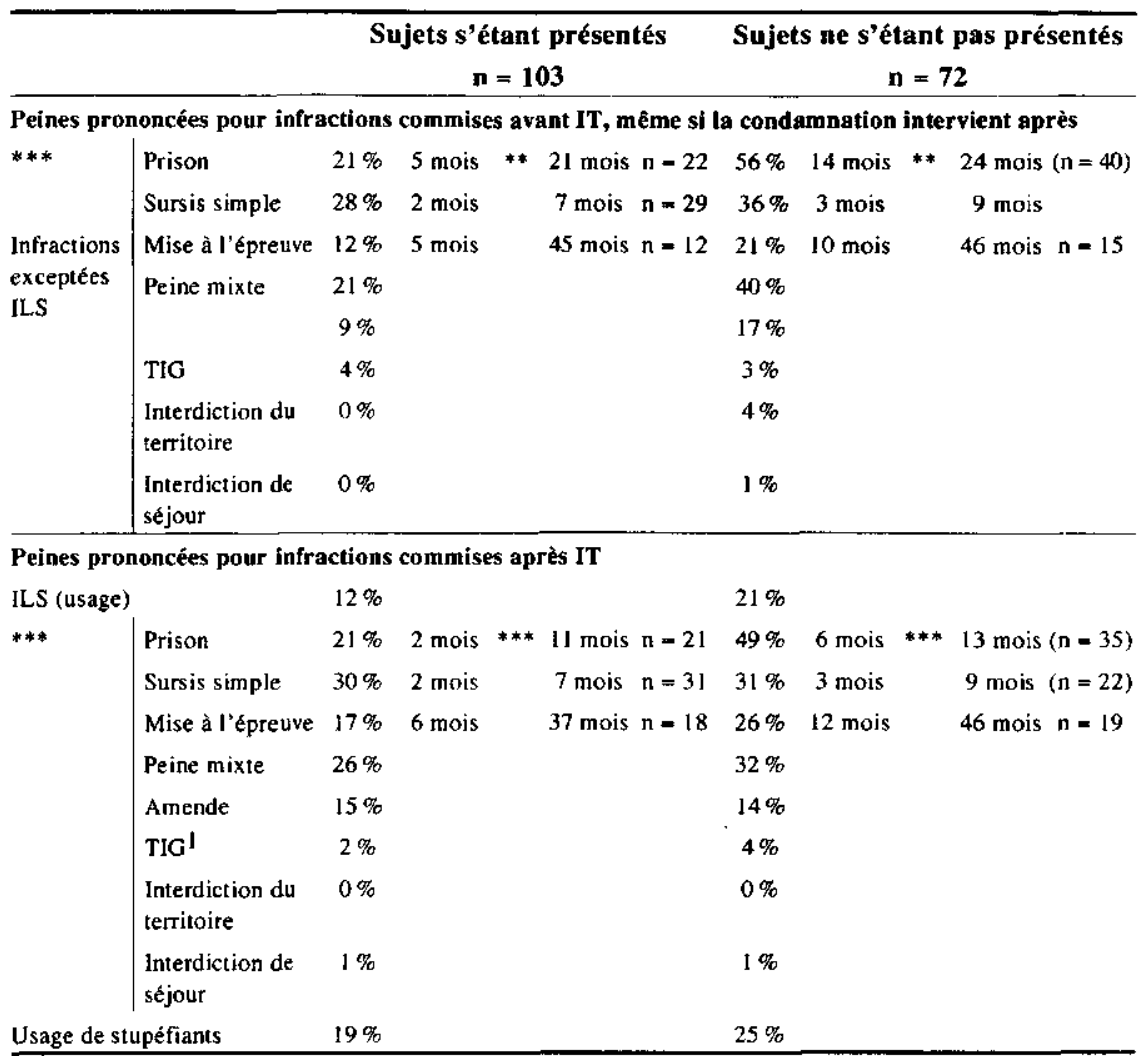

1. TIG : travaux d'intérêt général (travaux communautaires).

** $\quad \mathrm{p}<0,01$.

*** $\quad \mathrm{p}<0,001$

\section{Parcours judiciaires}

Une analyse comparative des sujets qui se sont présentés à l'injonction (groupe 1) et des sujets non venus (groupe 2) montre des différences quant au parcours antérieur et postérieur à l'injonction.

L'âge moyen des sujets étudiés est semblable : 25 ans. Parmi ceux-ci, on remarque que les femmes sont en proportion plus nombreuses dans le second groupe (non venues). Mais cette observation doit être tempérée par la faiblesse de l'échantillon (une dizaine). 


\section{Réserves méthodologiques}

La lecture des casiers judiciaires et l'approche progressive des techniques judiciaires ont révélé la nécessité de ne pas prendre en compte uniquement la date stricte de l'injonction pour analyser le passé et le devenir judiciaire du sujet.

Après plusieurs examens de délais susceptibles d'être retenus pour l'étude, il s'avère que c'est une période de 6 mois qui caractérise le mieux la prise en compte d'un effet êventuel de la mesure. Le délai correspond par ailleurs aux données de l'enquête nationale faite auprès des DDASS en 1990 (délai moyen de suivi des toxicomanes par les DDASS dans le cadre de l'IT).

Pour décrire les parcours avec plus de précision au niveau des comportements des sujets, on a considéré les dates des faits plutôt que celles des condamnations.

Situation antérieure à l'I.T. C'est sur le plan pénal que les différences significatives sont les plus marquées : les $3 / 4$ des sujets du groupe 2 ont commis des infractions avant l'injonction, contre $38 \%$ du groupe 1 .

Par ailleurs, on constate que plus de la moitié des sujets du groupe 2 font l'objet de peines d'emprisonnement $(56 \%)$, contre $21 \%$ pour les sujets $\mathrm{du} 1^{\mathrm{er}}$ groupe.

De même, les peines mixtes concernent $40 \%$ du $2^{\mathrm{e}}$ groupe contre $21 \%$ du premier. Les durées d'emprisonnement sont également différentes, plus longues pour les sujets du $2^{\mathrm{e}}$ groupe.

Ainsi les caractéristiques différenciant les groupes correspondent à la politique du parquet en matière de proposition d'injonction : celle-ci sera plutôt proposée à des personnes peu concernées par des problèmes antérieurs de drogue et/ou de délinquance. L'acceptation de la mesure semble plus répandue chez ces sujets.

Situation après l'injonction. Les peines de prison sont plus fréquentes (test significatif) pour le second groupe. La moitié des sujets ne s'étant pas présentés auront à subir ultérieurement une peine d'emprisonnement, contre seulement $20 \%$ parmi les sujets s'étant présentés à l'injonction.

Les durées d'emprisonnement sont encore plus importantes pour le $2^{\mathrm{e}}$ groupe.

En revanche, on n'observe pas de différence significative entre les deux groupes pour l'usage de stupéfiants (une tendance plus élevée est cependant indiquée pour le groupe 2 qui serait à confirmer sur un échantillon plus important). 


\section{IMPACT DE L'INJONCTION EN FONCTION DU PASSÉ JUDICIAIRE}

Suivi des sujets n'ayant pas commis de délit antérieur

Parmi eux, 64 sujets se sont présentés et 19 ne se sont pas présentés. Ils ont 24 ans en moyenne.

La différence est significative pour les peines de prison prononcées postérieurement à l'injonction, 4 fois plus fréquentes pour les sujets ne s'étant pas présentés.

Les mises à l'épreuve et peines mixtes sont aussi plus fréquentes.

\section{Suivi des sujets ayant commis des délits antérieurs}

Aucune différence significative n'apparaît dans le suivi des sujets. La notion de gravité de l'infraction peut être par contre approfondie pour tester l'efficacité de la mesure.

Pour les 32 sujets s'étant présentés à l'injonction et ayant commis des atteintes aux biens, 9 réitèrent les mêmes infractions 6 mois après.

En revanche, pour les sujets ne s'étant pas présentés, la poursuite de telles conduites caractérise 23 sujets sur 48 .

C'est la plus grande différence qui permet de mesurer le changement des conduites par rapport à l'I.T.

Pour les infractions diverses, les sujets concernés sont moins nombreux mais la tendance est la même : sur 7 sujets ayant commis antérieurement des infractions diverses, on en retrouve 3 en infractions ultérieures pour le groupe présenté, alors que la proportion est de 8 sur 10 pour le groupe qui ne s'est pas présenté.

\section{Analyse des parcours 6 mois après la date d'injonction}

Si, aux fins de l'analyse, on ne se situe plus à la date stricte de l'injonction mais dans un délai supplémentaire de 6 mois, les différences vont dans le même sens : la poursuite de la délinquance est plus marquée en moyenne pour les sujets qui ne se sont pas présentés à l'injonction. En revanche, l'usage de stupéfiants tendrait à la baisse, quel que soit le groupe, laissant presque supposer un effet strict de la garde à vue pour le $2^{\mathrm{e}}$ groupe, sinon un effet spécifique de l'injonction pour le $1^{\mathrm{er}}$ (mais les échantillons sont réduits et ne révèlent pas de différence significative).

On voit que l'évolution de la délinquance est beaucoup plus lourde pour les sujets ne s'étant pas présentés à l'injonction thérapeutique et n'ayant pourtant commis aucune infraction antérieure. Ainsi, pour les 92 sujets n'ayant pas commis d'ILS avant l'injonction, on relève 4 infractions 
6 mois après l'injonction. À l'inverse, pour les 59 sujets n'ayant pas commis d'ILS avant l'injonction et ne s'étant pas présentés, on relève 27 infractions, dont 1 ILS 6 mois après l'injonction.

On peut donc conclure que la mesure de l'injonction est associée à un impact positif quel que soit le passé judiciaire des sujets : on observe une diminution de la probabilité d'apparition d'actes délictueux tels qu'ils peuvent apparaître dans un casier judiciaire. Si pour les ILS, la proportion semble être la même, il convient toutefois d'observer que les effectifs sont faibles et que, par suite, toute interprétation doit être prudente.

\subsection{Place des centres spécialisés dans le suivi de l'injonction thérapeutique}

Sur un échantillon de 6111 toxicomanes consultants dans les centres spécialisés répartis dans la France entière, on observe que la part des sujets accueillis après injonction thérapeutique consentie est de $5 \%$ en moyenne ( $7 \%$ en région parisienne), ce qui indique une augmentation de ces patients depuis quelques années et une activité plus grande des équipes spécialisées en interaction avec les interventions judiciaires. L'installation des projets thérapeutiques dans les années 70 se fondait dans de nombreux cas sur la nécessité des démarches individuelles pour garantir la validité - et les chances de réussite - de la prise en charge. Aussi est-il intéressant de rechercher les caractéristiques de ces "nouveaux cas ", venant après injonction thérapeutique, en les comparant à ceux qui consultent sur demande individuelle.

\section{Aspects socio-familiaux}

La surreprésentation masculine est accentuée chez les sujets consultant après injonction thérapeutique $(86 \%)$; ils sont plus jeunes $(1 / 3$ ont moins de 25 ans et $9 \%$ ont moins de 20 ans), ils sont plus souvent célibataires et sans enfant $(69 \%)$. Ils habitent davantage chez leurs parents $(42 \%)$. Les décès parentaux sont moins fréquents (17\% des pères).

L'activité professionnelle est plus souvent continue (28\%). Les actifs sont plus souvent ouvriers $(57 \%)$. Ils bénéficient moins souvent du RMI ${ }^{6}$ (8\%).

\section{Aspects toxicologiques}

Le produit principal est majoritairement l'héroïne $(67 \%)$ - comme chez les autres consultants - toutefois on note plus souvent le cannabis $(23 \%)$. Les produits associés révèlent de la même façon la place prépondé-

6. Revenu minimum d' insertion. 
rante du cannabis dans $54 \%$ des cas, alors que la cocaïne $(8 \%)$, les médicaments $(10 \%)$, la codéine $(12 \%)$ et l'alcool $(20 \%)$ sont bien moins fréquents.

Les recours aux centres de soins et les sevrages antérieurs sont moins fréquents $(27 \%)$.

\section{Difficultés associées et comorbidité}

Les incarcérations antérieures sont fréquentes $(38 \%)$, parmi elles $20 \%$ ont eu lieu avant la toxicomanie. Au niveau de la psychopathologie, les tentatives de suicides sont moins fréquentes $(12 \%)$.

Le test VIH est moins souvent effectué $(59 \%)$, il est positif dans $31 \%$ des cas dépistés. les hépatites sont moins fréquentes (22\%).

Ainsi les sujets consultant après injonction thérapeutique semblent constituer un groupe de toxicomanes pour lesquels l'intervention judiciaire peut jouer un rôle d'incitation aux soins plus précoce que la motivation personnelle. Il resterait à comparer leur devenir thérapeutique à celui des autres consultants.

\subsection{Comparaison des toxicomanes incarcérés et des toxicomanes traités après injonction thérapeutique}

Un enjeu important apparaît à travers ces comparaisons de populations : l'intérêt d'un dépistage plus précoce se traduit-il par un accrochage aux soins et à une réinsertion d'autant plus solide?

À partir des éléments disponibles, une étude de suivi comparatif entre des sujets ayant une injonction et des sujets ne l'ayant pas, à difficultés judiciaires antérieures comparables, serait à encourager.

\section{LA SITUATION DU QUÉBEC}

Les données disponibles actuellement ne permettent pas de comparer adéquatement les situations qui prévalent actuellement en France et au Québec au niveau de l'intervention pénale s'adressant aux toxicomanes. Il est cependant possible de dresser un portrait global pour le Québec de l'intervention auprès du toxicomane judiciarisé.

Cette deuxième partie débutera par la présentation d'un bref rappel des lois canadiennes actuelles en matière de drogues. Bien que ces lois n'aient pas subi de modification capitale au cours des dernières décennies, il est possible d'observer de nouvelles tendances relatives aux accusations en matière de drogue, que nous exposerons brièvement. Bien que l'injonction thérapeutique ne soit pas vraiment appliquée au Québec, plusieurs contrevenants toxicomanes se retrouvent, au cours de leur processus judiciaire, 
dans un centre de réadaptation de la communauté environnante. Nous dresserons le portrait de ces personnes toxicomanes judiciarisées en vue de le comparer à celui des autres clients de ces centres de réadaptation et à celui des toxicomanes qui, eux, se retrouvent en détention.

\section{Bref rappel des lois canadiennes en matière de drogue}

Deux lois régissent les affaires de drogue au Canada. La Loi sur les stupéfiants et la Loi sur les aliments et drogues.

La Loi sur les stupéfiants contrôle la possession simple, le trafic, la possession en vue de trafic, l'importation et l'exportation, la culture, de mêrne que le délit d'ordonnance en ce qui concerne plus de 100 substances. Parmi les plus connues se retrouvent le cannabis, la cocaïne, la codéine, l'héroïne, la méthadone, la morphine et le phencyclidine. La possession, la culture et le délit d'ordonnance comportent des peines maximales ${ }^{7}$ de sept ans d'emprisonnement tandis qu'une peine d'incarcération à perpétuité est possible pour les autres délits. La Loi sur les stupéfiants s'intéresse également au profit tiré de la vente de ces drogues. Ainsi, la possession de biens ou de bénéfices excédant $1000 \$$ provenant de la vente de drogues peut entraîner une peine maximale de dix ans d'emprisonnement. Cette loi tente également d'attaquer les personnes qui cuvrent en vue de convertir, transporter, modifier, cacher, ou qui utilisent des biens obtenus par la transaction de ces drogues.

D'autre part, une deuxième loi canadienne, la Loi sur les aliments et drogues, comporte des dispositions concernant les psychotropes stimulants ou sédatifs utilisés à des fins médicales, et certaines substances psychoactives illicites (de façon générale les hallucinogènes). Ainsi, les psychotropes visés (entre autres les sédatifs hypnotiques non barbituriques et les tranquillisants mineurs) peuvent être vendus sous ordonnance. Néanmoins, la vente illicite de ces médicaments peut entraîner une peine maximale s'élevant à trois ans d'emprisonnement et/ou $5000 \$$ d'amende. Le trafic (et la possession en vue du trafic) des drogues contrôlées telles qu'amphétamines, barbituriques et quelques sédatifs et stimulants utilisés à des fins non médicales fait l'objet d'une peine maximale de dix ans d'emprisonnement. De plus, une première infraction pour un délit d'ordonnance est passible de trois années d'emprisonnement et/ou $5000 \$$ d'amende, tandis que la personne trouvée coupable de trafic (ou de possession en vue de trafic) est passible d'une peine maximale de dix ans d'emprisonnement. Enfin, la Loi sur les aliments et drogues prévoit également une catégorie

7. Nous ne rapportons ici que les peines maximales pouvant être imposées par voie de mise en accusation. Le tribunal peut également procéder par déclaration sommaire de culpabilité, les peines maximales pouvant être imposées s'avèrent alors moins importantes. 
d'infractions qui a trait aux profits de la vente de drogues. Les dispositions concernant les peines sont similaires à celles prévues par la Loi des stupéfiants.

\section{Tendances canadiennes en matière d'arrestations et de condamnations}

Au Canada, le nombre total d'accusations en vertu des lois sur les drogues dénote une progression légère et constante. On note toutefois certains déplacements importants en ce qui concerne les chefs d'accusation (moins d'affaires de simple possession):

[...] En 1981, 58838 délits de possession ont été déclarés, comparativement à 33037 en 1992. Entre 1977 et 1992, le nombre d'affaires relatives au trafic est passé de 10816 à 19539 , celui des affaires liées à l'importation, de 397 à 1073 , et celui des affaires liées à la culture, de 595 à 2 841. Dans l'ensemble, ces crimes représentaient $18 \%$ des affaires relatives à la drogue en 1977, et plus du double (42\%) de cette proportion en 1992 (Wolf et Reingold, 1994, p. 4-5);

On note également des différences concernant la nature des substances (moins d'affaires de cannabis).

Quatre-vingt-treize pour cent des délits de possession se rapportajent au cannabis en 1977, comparativement à $69 \%$ en 1992. À titre de comparaison, la proportion d'affaires relatives à la possession de cocaïne est passée de $1 \%$ en 1977 à $18 \%$ douze ans plus tard [...] le trafic de cocaïne constituait $37 \%$ des affaires de trafic en 1992, comparativement à seulement $4 \%$ en 1977. Par ailleurs, pendant la même période, la proportion d'affaires de trafic de cannabis a diminué, passant de $69 \%$ à $40 \%$ (Wolff et Reingold, 1994, p. 5).

Le nombre de personnes accusées en relation avec la cocaïne ou l'héroïne a donc fait des bonds importants au cours de la dernière décennie. La cannabis est toutefois demeuré la substance responsable du plus grand nombre de personnes accusées en vertu des lois sur les drogues au Canada ${ }^{8}$.

Pour traiter de ces dossiers, le ministère de la Justice, au Québec, a mis sur pied une équipe spécialisée constituée de 20 procureurs (Comité permanent de lutte aux drogues, 1994). Plus de la moitié (54\%) des condamnations pour affaires de drogues ont donné lieu à des peines de détention. Le tiers des personnes condamnées pour simple possession de drogues ont été incarcérées. Dans $40 \%$ de ces derniers cas, il s'agissait d'une période d'incarcération dépassant un mois (Santé et Bien-être social Canada, 1992).

8. Selon Riley et Oscapella (1995), plus de 500000 Canadiens ont des casiers judiciaires pour possession de cannabis. 
Il est possible de se questionner sur la pertinence de ces condamnations à cause desquelles les centres de détention canadiens accueillent plus de toxicomanes que les centres de réadaptation (Riley, 1994).

\section{La question de l'injonction thérapeutique}

Au moment où nous écrivons ces lignes (été 1996), le Québec n'utilise pas véritablement d'injonction thérapeutique envers ses toxicomanes. Des groupes de discussions (focus groups) tenus à l'automne 1994 et au printemps 1996 auxquels participaient, entre autres, des substituts du procureur de la Couronne de la région de Montréal, permettaient de constater que ces derniers ont l'impression d'avoir peu d'emprise pour inciter les accusés à entreprendre une démarche de réadaptation (Brochu et Drapeau, 1996; Schneeberger et Brochu, 1995a). Les détenus toxicomanes qui encourent de petites peines d'emprisonnement savent qu'ils seront libérés très rapidement compte tenu de la surpopulation actuelle des centres de détention. Ils n'ont pas la motivation nécessaire pour entreprendre une démarche thérapeutique qui sera nécessairement plus longue que leur période d'incarcération. Par contre, au niveau de la défense, la perception est différente et on conseille assez fréquemment aux clients qui semblent éprouver des problèmes face à la drogue de suivre une thérapie. Bien sûr, pour le client, le renvoi vers un centre de traitement est souvent perçu comme une façon d'échapper à une éventuelle peine d'emprisonnement. En somme, à la suite d'une évaluation des problèmes de toxicomanie de la personne accusée s'il y a entente entre les parties, le juge pourra opérer un renvoi dans un centre de réadaptation pour toxicomanes. Il suspendra alors sa sentence jusqu'à la fin du traitement. Dans le cas où le traitement s'avère un succès, il pourra réduire sa sentence (sursis ou ordonnance de probation) tout en ajoutant possiblement la condition de poursuivre la démarche de traitement sur une base externe. Si le contrevenant abandonne la cure sans entente préalable, un mandat d'arrestation est émis pour manquement à une condition de remise en liberté. Des rencontres avec des juges et des agents de probation permettent de croire qu'une année de thérapie équivaut généralement à deux ans de réduction de peine (Schneeberger et Brochu, 1995a). Cette façon de procéder permet au tribunal de suivre le dossier tout en s'assurant que le traitement est effectivement suivi. Elle a toutefois été critiquée par les tribunaux supérieurs car 1) elle transforme le juge en agent de probation qui veille, par des rencontres périodiques avec le contrevenant, au bon déroulement de la démarche, et 2) elle retarde indûment le prononcé de la sentence (voir Brochu et Drapeau, 1996, pour une discussion sur ce thème).

La loi C-22 modifiant le Code criminel en matière de détermination de la peine, sanctionnée le 13 juillet 1995 devrait être mise en application à l'automne 1996. Cette loi veut modifier la pratique actuelle des tribunaux alors que l'incarcération est présentée comme une mesure de dernier 
recours. On favorise ainsi l'utilisation de mesures substitutives à l'emprisonnement. Pour certains contrevenants dont le délit nécessite une peine d'incarcération de moins de deux ans, on propose au tribunal d'utiliser l'emprisonnement avec sursis tout en assortissant cette mesure d'un renvoi vers un centre de traitement, lorsque cela peut s'avérer approprié. Dans ces circonstances, le juge prononce la peine, mais sursoit à son exécution tant et aussi longtemps qu'il n'y a pas de manquement à l'ordonnance. On évite ainsi les délais déraisonnables avant le prononcé de la sentence; on enlève au juge la tâche d'agent de surveillance qu'il avait parfois revêtue. La sentence prononcée pourrait également constituer une pression supplémentaire pour que le contrevenant se plie aux exigences de la démarche de réadaptation (Brochu et Drapeau, 1996).

Cela amène à se questionner sur la notion de volontariat du contrevenant lors du renvoi vers un centre de traitement. Le nouveau texte de loi n'est pas clair. Ainsi, il est spécifié que, lorsqu'il s'agit d'une ordonnance d'emprisonnement avec sursis, le tribunal peut joindre aux conditions obligatoires celle de «suivre un programme de traitement approuvé par la province $»$. On ne mentionne pas la nécessité du consentement du contrevenant ou du directeur du programme de traitement. Dans les faits, tous les acteurs pénaux rencontrés croient fermement qu'une telle mesure ne peut être couronnée de succès que dans la mesure où cette démarche est souhaitée par la personne concernée au premier chef (Brochu et Drapeau, 1996).

Somme toute, il n'existe pas véritablement d'injonction thérapeutique dans la pratique actuelle des tribunaux canadiens. Toutefois, la cour sait exercer certaines pressions, plus ou moins subtiles, de façon à encourager la motivation de certains contrevenants pour qu'ils entreprennent une démarche de réadaptation supervisée par un centre de traitement. Cette influence se fera-t-elle encore plus contraignante avec l'application de la nouvelle loi modifiant la détermination de la peine? Les acteurs pénaux en doutent; seul l'avenir nous apportera la véritable réponse.

\section{Profil de la clientèle judiciarisée dans les centres de réadaptation}

Compte tenu des négociations pré-sentencielles actuelles, entre 16 et $21 \%$ de la clientèle de différents centres publics de réadaptation en toxicomanie au Québec se trouvent en instance d'inculpation, de procès ou de sentence au moment de la demande d'aide (Brunelle, 1994; Schneeberger et Brochu, 1995b). Une étude estime à près de 10\% la clientèle qui se présente en traitement à la suite des recommandations des représentants du système judiciaire ou parajudiciaire (Laflame-Cusson, Guyon et Landry, 1994).

La comparaison des profils des personnes judiciarisées et non judiciarisées des centres publics de réadaptation permet de constater que les per- 
sonnes aux prises avec des problèmes judiciaires sont majoritairement de jeunes hommes célibataires peu intégrés au marché de l'emploi (Schneeberger et Brochu, 1995b). Bien plus, le profil bio-psycho-social des toxicomanes judiciarisés, tel que mesuré par l'Indice de gravité d'une toxicomanie $(\mathrm{IGT})^{9}$ ) est, de façon générale, plus détérioré que celui des personnes qui n'ont jamais connu de problèmes avec la justice (Guyon et Brochu, 1994). Enfin, leur démarche de réadaptation se caractérise par une moins longue persistance en traitement. Toutefois à persévérance égale, celui-ci se révèle aussi bénéfique que pour l'ensemble de la clientèle (Beaudet, Brochu, Bergeron et Landry, 1995). Devant ces constatations, un important centre public de réadaptation de la toxicomanie a mis sur pied un programme spécialisé pour le traitement des personnes toxicomanes éprouvant des problèmes avec la justice. Plus spécifiquement, ce programme, s'adressant tout particulièrement aux délinquants persistants (voir Fréchette et Leblanc, 1987), tentera de motiver ces personnes à demeurer en traitement et à abandonner leur style de vie déviant (Brochu, 1995).

\section{Les toxicomanes en détention}

Le cheminement le plus usuel pour un contrevenant toxicomane demeure encore la prison ou le pénitencier. Plusieurs études québécoises ont tenté de déterminer la prévalence de consommateurs et de toxicomanes au sein des diverses populations carcérales du Québec. Ces études nous informent que plus de la moitié des détenus avait fait usage d'une substance psychoactive illicite au cours du mois ayant précédé leur incarcération (Brochu et al., 1992; Lévesque, 1994; Service correctionnel du Québec, 1995). De plus, les différentes études estiment généralement que le tiers de la population admise et la moitié de la population résidant ${ }^{10}$ dans les institutions carcérales au Québec ${ }^{11}$ éprouvent des problèmes de toxicomanie suffisamment importants pour nécessiter un traitement (Brochu et Guyon, 1994a; Forget, 1990, Hodgins et Côté, 1990 et 1991; Lévesque, 1994; Schneeberger et Brochu [sous presse]). Dans bien des cas, le profil bio-

9. L'IGT constitue une validation québécoise de l'Addiction Severity Index (McLellan, Luborsky et O'Brien, 1980) effectuée par Bergeron et al. (1992) pour une clientèle toxicomane et par Brochu et Guyon (1995) pour les personnes toxicomanes judiciarisées.

10. Il faut comprendre ici que la population résidente présente généralement un profil délinquant plus important que la population admise. Ainsi, dans un centre de détention provincial, la population admise est constituée en grande partie par de très courtes sentences reliées à des non-paiement d'amende ou d'autres délits de très faible gravité, alors que la population résidente est constituée de personnes devant purger de plus longues sentences pour des délits de gravité supérieure.

11. Autant les pénitenciers fédéraux qui accueillent les personnes condamnées à des peines de deux ans et plus que les prisons provinciales qui détiennent les personnes condamnées à des peines de moins de deux ans. 
psycho-social tel que mesuré par l'IGT se trouve plus détérioré que chez les toxicomanes admis en centre public de réadaptation pour toxicomanie (Brochu et Guyon, 1994b).

\section{Les programmes de traitement de la toxicomanie en détention}

Deux modèles guident la disposition des programmes de traitement de la toxicomanie dans les services correctionnels. Ainsi, le Service correctionnel du Canada, qui gère de longues peines d'incarcération (deux ans et plus) a mis sur pied un continuum de services en toxicomanie dont il assure la responsabilité. Ce continuum va de l'évaluation des problèmes de toxicomanie lors de l'admission en pénitencier à la référence vers des programmes de prévention (primaire ou secondaire selon les besoins identifiés), en passant par les programmes de communautés thérapeutiques pour les personnes nécessitant une prise en charge longue et intensive (Service correctionnel du Canada, 1991).

Le service correctionnel du Québec (responsable de la gestion des sentences de détention de moins de deux ans) a préféré opter pour la prestation de services dans l'environnement naturel de la personne. Un protocole d'entente entre le ministère de la Santé et des Services sociaux et le ministère de la Sécurité publique a donc été signé en 1989. Il garantit aux personnes judiciarisées des services adéquats en matière de santé, notamment en toxicomanie. Toutefois, le contexte propre au milieu carcéral, la crainte qu'entretiennent parfois certains centres de réadaptation vis-à-vis de cette clientèle, et les caractéristiques particulières des toxicomanes judiciarisés ont empêché, jusqu'à aujourd'hui, cette équité soutenue dans l'entente interministérielle (Schneeberger et Brochu, 1995a).

Un comité d'experts s'est alors réuni en 1994-1995 en vue de rédiger un ensemble de rapports traitant de la situation actuelle et d'élaborer certaines recommandations. Au nombre de ces recommandations ${ }^{12}$, notons (1) la mise sur pied d'un comité de travail chargé de l'élaboration et du suivi d'un plan de coordination des services; (2) l'élaboration d'un cadre de référence traitant des zones de responsabilités respectives de chacun, de la confidentialité des informations colligées, des modalités de collaboration interréseaux, des mécanismes de communication ; (3) la mise sur pied de séances de formation d'appoint par et pour les membres du personnel des réseaux impliqués; et (4) la facilitation de l'accès aux services de réadaptation pour les usagers contrevenants (Brochu et al., 1995). Ces recommandations ont pour objectif d'offrir l'accès à des services de réadaptation adéquats aux personnes toxicomanes, quel que soit leur statut judiciaire.

12. Vingt-trois recommandations ont été déposées. 


\section{CONCLUSION}

Dans nos deux pays, on observe des phénomènes similaires d'augmentation des conduites de délinquance identifiées en relation avec des usages et trafics de drogues, le cannabis étant le produit le plus désigné dans les statistiques policières, puis la cocaīne pour l'Amérique du Nord et l'héroïne pour la France. Des difficultés sont rencontrées pour comparer l'ensemble des statistiques policières et judiciaires: au-delà des biais classiques liés aux fluctuations d'activités, les définitions entre usagers et toxicomanes ne sont pas toujours bien délimitées, ainsi les interpellations en France ont augmenté de $13 \%$ en 1994, en liaison surtout avec des affaires de trafic, sans qu'on puisse parler d'augmentation du nombre de toxicomanes.

Les deux contextes législatifs ne prévoient pas les mêmes sanctions face à des usages de produits illicites, et leur utilisation montre des pratiques différentes: le recours à l'incarcération apparaît plus fréquent au Canada, où plus de la moitié des condamnations pour affaires de drogues ont donné lieu à des peines de détention et où plus du tiers des personnes condamnées le sont pour simple possession (contre $9 \%$ en France).

Même si dans les deux pays, on relève des activités importantes de répression et d'incarcération face aux toxicomanes, les orientations françaises vers des interventions plus sanitaires méritent qu'on s'interroge sur leur efficacité dans une perspective générale de solution de remplacement à l'incarcération.

Au niveau de la méthodologie d'enquête utilisée, l'absence de registre de patients en France empêche la constitution d'échantillons représentatifs de clientèles des centres de soins et compromet la possibilité d'études longitudinales sur des groupes comparatifs: toxicomanes incarcérés, toxicomanes bénéficiant d'une injonction de soins, toxicomanes traités sur leur seule motivation. Aussi les postes d'observation utilisés pour mesurer l'impact des injonctions dépendent-ils des services de justice et de soins; les informations recueillies peuvent alors souffrir d'un certain nombre de biais, en liaison avec le fonctionnement de ces services. Ainsi l'évolution de statistiques d'activités ne reflète pas forcément celle de la population toxicomane et la mise en place d'enquêtes spécifiques est indispensable, dans un contexte d'indépendance de la recherche.

Au niveau des résultats obtenus dans des études nationales, même si l'injonction thérapeutique évite à un nombre croissant d'usagers de drogues l'incarcération, toutefois des limites sont rencontrées : cette mesure est proposée de façon sélective, sur des critères sociaux et judiciaires plus favorables que les caractéristiques moyennes des toxicomanes, elle n'est acceptée et suivie que par la moitié environ des sujets. De plus, parmi les toxicomanes incarcérés, presque 1 sur 5 a déjà bénéficié d'une injonction 
(et $28 \%$ des héroïnomanes incarcérés) : Ce taux reflète en partie l'échec de l'injonction au moment de ces prises en charge (mais pas dans un parcours global) : un seuil d'efficacité de l'injonction face aux risques de récidive est ainsi identifié.

L'enquête spécifique menée dans un département de la région parisienne où les activités face à la toxicomanie sont particulièrement nombreuses au niveau des interpellations, des incarcérations, des injonctions thérapeutiques et des prises en charge spécialisées montre, sur un échantillon de 175 sujets, le rôle de l'injonction : celle-ci apparaît significativement positive pour ceux qui la suivent, par rapport à ceux qui la refusent, au niveau du devenir judiciaire. Ces résultats suggèrent alors un rôle de l'injonction dans la prévention de la récidive. Toutefois, les changements de comportement d'intoxication, même s'ils vont dans un sens d'amélioration, ne montrent pas de différence significative. Faudrait-il constituer un échantillon plus important de sujets suivis en approfondissant ce thème : conservent-ils leurs habitudes d'usages de stupéfiants en évitant mieux les interpellations? Changent-ils de mode de vie associé à la toxicomanie, et qu'en est-il sur le plan de la santé ?

Ces résultats, obtenus sur un échantillon partiel certes, incitent tout de même à aborder le rôle d'extension du contrôle social que pourrait avoir l'injonction thérapeutique pour un certain nombre de sujets peu exposés aux risques d'incarcération, sous prétexte de favoriser une relation clinique possible après l'injonction.

Les interrelations entre les domaines du soin et de la justice sont soumises à des contraintes de confidentialité des données individuelles et à des missions de santé publique : favoriser l'accès aux soins des individus tout en préservant la sécurité et l'état de santé de la collectivité. Il peut y avoir divergence entre l'efficacité individuelle à moyen terme dans le trajtement de la toxicomanie et l'appréciation globale instantanée d'une mesure collective, dans son bilan économique et dans la surcharge de réseaux des professionnels des soins et de la justice, qui n'ont pas toujours l'habitude de travailler en commun.

Il n'en demeure pas moins que face aux problèmes accrus que rencontre une population jeune sur le plan des comportements à risque, comme l'usage des drogues, et sur le plan de l'adaptation sociale, des réseaux de professionnels doivent être encouragés et notamment entre les domaines de l'intervention judiciaire et sanitaire. Une mesure comme l'injonction thérapeutique en est une expression tout à fait intéressante, même si elle comporte naturellement des limites. 


\section{BIBLIOGRAPHIE}

BAUDET N., BROCHU S., BERGERON J., LANDRY M., Persistance en traitement et impact du processus de réadaptation des personnes toxicomanes judiciarisées admises à Domrémy-Montréal. Rapport n 5, 1995, Université de Montréal : Centre international de criminoiogie comparée.

BOUCAUD, P., Le traitement de la pharmacodépendance en droit comparé. Cahier thématique, 1990, 1, (27-40).

BROCHU, S., Drogue et criminalité : mythe ou réalité ?, Montréal : Centre international de criminologie comparée, (1994).

BROCHU, S., Drogues et questions criminelles. Montréal : Presses de l'Université de Montréal (1995).

BROCHU, S., Drogues et criminalité : une relation complexe. Montréal : Presses de l'Université de Montréal (1995).

BROCHU, S., DESJARDINS, L., DOUYON, A., FORGET, C., Drug use prevalence among offenders. In : LOSEL, F., BENDER, D., BLIESNER T. (eds). Psychology and Law : International Perspectives, (pp 105-110). Berlin : Walter de Gruyter (1992).

BROCHU, S., DRAPEAU, A., La pratique des tribunaux face aux renvois vers les centres de traitement de la toxicomanie. Montréal : Portage (1996).

BROCHU, S., GUYON, L., Drug addiction Among Inmates. Fourth european conference of law and psychology : Barcelone, avril 1994, (1994a).

BROCHU, S., GUYON, L., Profil de populations toxicomanes adultes: DomrémyMontréal et Centre de détention de Montréal. Symposium organisé dans le cadre du $25^{\mathrm{e}}$ anniversaire du Centre de réadaptation Alternatives, Montréal, 1931, octobre, (1994b).

BROCHU, S., SCHNEEBERGER, P., La réadaptation des personnes toxicomanes judiciarisées. Rapport $n^{\circ} 4$. Université de Montréal : Centre intemational de criminologie comparée (1995).

BROCHU, S., SCHNEEBERGER, P., BARIL, R., BASTIEN, L., BOULARD, M., CLOUTIER, R., COUTURE, P., DUFRESNE, , ., FORGET, J., GODIN, G., LEVESQUE, M., MENARD, P., TOPP, J., VERREAULT, S., Recommandations concernant les services à offrir aux personnes présentant une double problématique toxicomanie/délinquance. Rapport $n^{\circ} 6$. Université de Montréal : Centre internationâi de criminologie comparée (1995).

BRUNELLE, N., Analyse biopsychosociale de personnes admises en traitement pour la toxicomanie. Université de Montréal : mémoire de maîtrise, 1994.

COMITÉ PERMANENT DE LUTTE AUX DROGUES, La coordination des actions gouvernementales en matière de lutte aux drogues. Sainte-Foy. Les publications du Québec, 1994.

DAMADE, M., Les réponses sociales à l'usage des drogues et de l'alcool. Bull. CLCJ, $1989 ; 18,(85-88)$.

DELOBEL, B., Usage de stupéfiants et alternatives à l'incarcération. Rapport Rech. DEA Droit Pénal, Sci. Criminelles, Fac. droit Bordeaux I, 1990, 96 p.

DELPUECH, D., Le juge et le toxicomane. Trimestre Psychanalytique, 1989, 4; (6777). 
FACY, F., Description de l'activité des services chargés notamment du suivi des mesures d'injonction thérapeutique 1989-1990. Rapport DGS-INSERM, 1991, 56 p.

FACY, F., TASSIN, J. P., Drogues et toxicomanie. INSERM-NATHAN, 1993, 100 p.

FAUCONNET, L., Le contrôle judiciaire socio-éducatif, Interventions. 1986; 8, (1618).

FORGET, C., La consommation de substances psycho-actives chez les détenus du centre de détention de Montréal. Université de Montréal : Mémoire de mâtrtise inédit, 1990.

FRÉCHETTE, M., LEBLANC, M., Délinquances et délinquants. Chicoutimi : Gaétan Morin, 1987.

GUYON, L., BROCHU, S., Addiction and Criminal Background in Rehabilitation Centers for Drug and Alcohol Abuser Clientele, Montréal : Recherche et intervention sur les substances psychoactives-Québec (RISQ), 1994.

HODGINS, S., CÔTÉ, G., Prévalence des troubles mentaux chez les détenus des pénitenciers du Québec. Santé mentale au Canada, 38, 1-5, 1990.

HODGINS, S., CÔTÉ, G., The mental health of penitentiary Inmates in Isolation. Canadian Journal of Criminology, 33, (2), 175-182, 1991.

LAFLAMME-CUSSON, S., GUYON, L., LANDRY, M., Analyse comparée de la clientèle de trois centres de réadaptation pour personnes alcooliques et toxicomanes à partir de l'IGT. Montréal : RISQ, 1994.

LEBLANC, S., De l'usage du toxicomane ou fondements de la loi Mazeaud. Doct. Pharmacie, Univ. Poitiers, $1991 ; 79$ p.

LEROY, B., L'Europe des douze face à la demande de stupéfiants. Étude comparative des législations et des pratiques judiciaires. Commission des Communautés Européennes, CEC/LUX/V/E/1/28/91, 1991.

LÉVESQUE, M., La criminalité et la consommation de drogues : une double problématique (255-272). In : P. BRISSON (Éd.). L'usage des drogues et la toxicomanie, vol. 2. Bourcherville : Gaétan Morin, 1994.

LIGIER, D., L'échec du législateur. Esprit, 1980; 11-12, (127-136).

ORS ÎLE-DE-FRANCE, L'injonction thérapeutique. In : Le phénomène toxicomanie dans les Hauts-de Seine. Première approche. ORS, Mai 1991, (49-5I).

PARQUET, Ph. J., BAILLY, D., Législation et pharmacodépendance. Neuro-psy, 1988,$3 ; 2,(85-94)$.

PELLETIER, M., Mission d'étude sur l'ensemble des problèmes de la drogue. La Documentation française, $1978 ; 283 \mathrm{p}$.

PORTER, L., ARIF, A. E., CURRAN, W. J., La loi et le traitement de la pharmacodépendance et de l'alcoolodépendance. Étude comparative des législations existantes. OMS, Genève, 1988, 227 p.

RILEY, D., La réduction des méfaits liés aux drogues : politique et pratique. In: P. BRISSON (Éd.). L'usage des drogues et la toxicomanie (pp. 129-147), 1994. Chicoutimi : Gaétan Morin.

SABATINI, J., À propos du caractère thérapeutique de l'injonction de soins. Interventions, 1990, mai, (179-180).

SCHNEEBERGER, P., BROCHU, S., Alcool, drogues illicites et criminalité : étude d'une population incarcérée à l'établissement de détention de Trois-Rivières. Psychotropes : Revue internationale sur les toxicomanies, 1, (1), 1995. 
SCHNEEBERGER, P., BROCHU, S., Profil des activités délictueuses chez les toxicomanes en traitement : rapport $n^{\circ} 2$. Université de Montréal : Centre international de criminologie comparée, 1995.

SERVICE CORRECTIONNEL DU CANADA, Rapport du groupe d'étude sur la réduction de la toxicomanie. Ottawa: Approvisionnements et services Canada, 1991.

SERVICE CORRECTIONNEL DU QUÉBEC, Portrait de la clientèle correctionnelle du Québec. Sainte-Foy : ministère de la Sécurité publique, 1995.

TIMBART, O., L'usage de stupéfiants dans les condamnations. Infostat Justice, 1994, $n^{\circ} 38: 1-4$. 